\title{
TWO-WEIGHTED CRITERIA FOR INTEGRAL TRANSFORMS WITH MULTIPLE KERNELS
}

\author{
VAKHTANG KOKILASHVILI \\ International Black Sea University \\ 2, Agmashenebeli Alley 13th km., Tbilisi 0131, Georgia \\ E-mail: kokil@rmi.acnet.ge \\ ALEXANDER MESKHI \\ A. Razmadze Mathematical Institute, Georgian Academy of Sciences \\ 1, M. Aleksidze St., 0193 Tbilisi, Georgia \\ Current address: \\ Scuola Normale Superiore \\ Piazza dei Cavalieri 7, 56126 Pisa, Italy \\ E-mail:a.meskhi@sns.it
}

\begin{abstract}
Necessary and sufficient conditions governing two-weight $L^{p}$ norm estimates for multiple Hardy and potential operators are presented. Two-weight inequalities for potentials defined on nonhomogeneous spaces are also discussed. Sketches of the proofs for most of the results are given.
\end{abstract}

Introduction. The survey deals with the boundedness (compactness) criteria for a large class of classical integral operators in classical Lebesgue spaces. For the considerable achievement in this area we refer to [GR], [K2], [OK], [GGKK], [BK], [KP], [GM], $[\mathrm{EKM}]$. The latter book focuses our attention on boundedness (compactness) criteria of integral operators arising naturally in boundary value problems for PDE, the spectral theory of differential operators, continuum and quantum mechanics, stochastic processes, etc.

The paper is organized as follows: in Section 1 we present some well-known two-weight results for the classical Hardy and potential operators; weighted boundedness criteria for

2000 Mathematics Subject Classification: 47G10, 26A33, 31B15, 46E30.

Key words and phrases: potential with multiple kernels, higher dimensional Hardy operators, two-weight inequalities, nonhomogeneous spaces.

Research of the first author supported by KBN grant $00-000$.

The paper is in final form and no version of it will be published elsewhere. 
Volterra-type integral operators with multiple kernel are discussed in Section 2; Section 3 is devoted to the new two-weight conditions guaranteeing the boundedness of the double Hardy operators; two-weight norm inequalities for Riesz potentials with multiple kernels are established in Section 4; an extension of Stein-Weiss theorem for fractional integrals defined on nonhomogeneous spaces is given in Section 5 .

1. Preliminaries. Let $X$ be a space with a complete measure $\mu$. Suppose that $w$ is a nonnegative locally $\mu$-integrable function on $X$. Such functions are called weight functions. By $L_{w}^{p}(X)(1 \leq p<\infty)$ we denote the set of all $\mu$-measurable functions $f$ for which the norm

$$
\|f\|_{L_{w}^{p}(X)}=\left(\int_{X}|f(x)|^{p} w(x) d \mu(x)\right)^{1 / p}<\infty .
$$

The criterion for the two-weight inequality for the Riesz potential

$$
I_{\alpha} f(x)=\int_{R^{n}} \frac{f(y)}{|x-y|^{n-\alpha}} d y, \quad 0<\alpha<n,
$$

has been given by the following (see $[\mathrm{KK}]$, [GGKK], [SWZ]).

Theorem A. Let $1<p<q<\infty$. Then $I_{\alpha}$ is bounded from $L_{w}^{p}\left(R^{n}\right)$ into $L_{v}^{q}\left(R^{n}\right)$ if and only if

$$
\sup _{\substack{x \in R^{n} \\ r>0}}(v B(x, 2 r))^{1 / q}\left(\int_{|x-y|>r}|x-y|^{(\alpha-n) p^{\prime}} w^{1-p^{\prime}}(y) d y\right)^{1 / p^{\prime}}<\infty
$$

and

$$
\sup _{\substack{x \in R^{n} \\ r>0}}\left(w^{1-p^{\prime}} B(x, 2 r)\right)^{1 / p^{\prime}}\left(\int_{|x-y|>r}|x-y|^{(\alpha-n) p} v(y) d y\right)^{1 / q}<\infty .
$$

These criteria avoid the notion of capacity and can be easily verified.

The proof of Theorem A is based on the two-weight weak-type criterion for the Riesz potentials given by E. Sawyer [Sa1] and on the more transparent one established by M. Gabidzashvili [Ga], [GK] (see also [KK]).

For a solution of the two-weight problem for integral transforms with positive kernels we refer to $[\mathrm{GGKK}]$ and [SWZ]. The results mentioned above were essentially employed, for example, in the theory of the spaces of differentiable functions (see [B1]-[B3]).

In our opinion, one of the challenging problems in the weight theory currently is to solve the two-weight problem for integral transforms with the product kernels. It is worth mentioning that this problem remains still open.

Let us recall two-weight criteria for the one-sided potentials:

$$
R_{\alpha} f(x)=\int_{0}^{x} f(t)(x-t)^{\alpha-1} d t ; \quad W_{\alpha} f(x)=\int_{x}^{\infty} f(t)(t-x)^{\alpha-1} d t
$$

where $\alpha>0$. If $\alpha=1$, then $R_{\alpha}$ is the classical Hardy operator defined by

$$
H f(x)=\int_{0}^{x} f(t) d t
$$

The two-weight problem for $H$ have been solved by B. Muckenhoupt [Mu1] for $p=q$, and F. J. Bradly [Br], V. Kokilashvili [K1] for $p \leq q$ (see also [Ma], Ch. 1), while necessary 
and sufficient conditions on weights guaranteeing the boundedness of $R_{\alpha}$ with $\alpha>1$ from $L_{w}^{p}\left(R_{+}\right)$to $L_{v}^{q}\left(R_{+}\right)$have been established by V. Stepanov [St1] and J. Martin-Reyes and E. Sawyer [MS]. Namely, the following statement holds:

Theorem B. Let $1<p \leq q<\infty, \alpha \geq 1$. Then the operator $R_{\alpha}$ acts boundedly from $L_{w}^{p}\left(R_{+}\right)$to $L_{v}^{q}\left(R_{+}\right)$if and only if the following two conditions

$$
\begin{gathered}
A_{1}:=\sup _{t>0}\left(\int_{t}^{\infty} \frac{v(x)}{(x-t)^{(1-\alpha) q}} d x\right)^{1 / q}\left(\int_{0}^{t} w^{1-p^{\prime}}(x) d x\right)^{1 / p^{\prime}}<\infty ; \\
A_{2}:=\sup _{t>0}\left(\int_{t}^{\infty} v(x) d x\right)^{1 / q}\left(\int_{0}^{t} \frac{w^{1-p^{\prime}}(x)}{(t-x)^{(1-\alpha) p^{\prime}}} d x\right)^{1 / p^{\prime}}<\infty
\end{gathered}
$$

hold. Moreover, there exist positive constants $c_{1}$ and $c_{2}$ depending only on $\alpha, p$ and $q$ such that $c_{1} \max \left\{A_{1}, A_{2}\right\} \leq\left\|R_{\alpha}\right\| \leq c_{2} \max \left\{A_{1}, A_{2}\right\}$.

For a survey of the results on the boundedness and compactness problems for $R_{\alpha}$ in the case when the order of integration is not less than one see [St2]. For the same problems for one-sided potentials defined on the real line we refer to [KM1].

K. Andersen and E. Sawyer $[\mathrm{AS}]$ proved the following

Theorem C. Let $1<p<q=p /(1-\alpha p), 0<\alpha<1 / p$. Then the inequality

$$
\left\|u R_{\alpha} f\right\|_{L^{q}\left(R_{+}\right)} \leq c\|u f\|_{L^{p}\left(R_{+}\right)}
$$

holds if and only if

$$
\sup _{\substack{a, r \\ 0<r<a}} \frac{1}{r}\left(\int_{a}^{a+r} u^{q}(x) d x\right)^{1 / q}\left(\frac{1}{r} \int_{a-r}^{a} u^{-p^{\prime}}(x)\right)^{1 / p^{\prime}}<\infty .
$$

Further, in the case of more general exponents $p$ and $q$ we have

TheOrEm D ([EKM], p. 131). Let $1<p<q<\infty$. Suppose that $0<\alpha<1 / p$. Then $R_{\alpha}$ is bounded from $L^{p}\left(R_{+}\right)$to $L_{u}^{q}\left(R_{+}\right)$if and only if

$$
C_{1} \equiv \sup _{\substack{h, a \\ 0 \leq h \leq a}}\left(\int_{a}^{a+h} u(x) d x\right)^{1 / q} h^{\alpha-1 / p}<\infty .
$$

Moreover, there exist positive constants $c_{k}, k=1,2$ depending only on $\alpha, p$ and $q$ such that $c_{1} C_{1} \leq\left\|R_{\alpha}\right\| \leq c_{2} C_{1}$.

From the previous theorem we obtain the following

Corollary A. Let $0<\alpha<1 / p$ and let $q=p /(1-\alpha p)$. Then the following conditions are equivalent:

(i) $R_{\alpha}$ is bounded from $L^{p}\left(R_{+}\right)$to $L_{v}^{q}\left(R_{+}\right)$;

(ii) $\|v\|_{L^{\infty}\left(R_{+}\right)}<\infty$.

For the latter result see [PS].

TheOREM E ([M1]). Let $1<p \leq q<\infty$ and let $\alpha>1 / p$. Then the following conditions are equivalent:

(i) $R_{\alpha}$ is bounded from $L^{p}\left(R_{+}\right)$to $L_{v}^{q}\left(R_{+}\right)$; 
(ii)

$$
C_{2} \equiv \sup _{a>0} C_{2}(a) \equiv\left(\int_{a}^{\infty} v(x) x^{(\alpha-1) q} d x\right)^{1 / q} a^{1 / p^{\prime}}<\infty
$$

(iii)

$$
C_{3} \equiv \sup _{k \in Z} C_{3}(k) \equiv \sup _{k \in Z}\left(\int_{2^{k}}^{2^{k+1}} v(x) d x\right)^{1 / q} 2^{k(\alpha-1 / p)}<\infty .
$$

Moreover, $\left\|R_{\alpha}\right\| \approx C_{2} \approx C_{3}$.

The next statement gives the compactness criteria for $R_{\alpha}$.

Theorem $\mathrm{F}([\mathrm{M} 1])$. Let $1<p<\infty$ and let $\alpha>1 / p$. Then the following conditions are equivalent:

i) $R_{\alpha}$ is compact from $L^{p}\left(R_{+}\right)$to $L_{v}^{q}\left(R_{+}\right)$;

ii) $\lim _{a \rightarrow 0} C_{2}(a)=\lim _{a \rightarrow \infty} C_{2}(a)=0$;

iii) $\lim _{k \rightarrow+\infty} C_{3}(k)=\lim _{k \rightarrow-\infty} C_{3}(k)=0$.

For the last two statements see also [EKM] (Section 2) and [Pr]. When $p=q=2$ the assertion was obtained in [NS].

In the diagonal case $p=q$ the two-weighted characterization for the Riesz potentials has been done in [MV], [VW].

For one-sided potentials we have

Theorem G ([KM2]). Let $1<p<\infty$ and let $0<\alpha<1 / p$. Then the operator $R_{\alpha}$ is bounded from $L^{p}\left(R_{+}\right)$to $L_{v}^{p}\left(R_{+}\right)$if and only if $W_{\alpha} v \in L_{l o c}^{p^{\prime}}\left(R_{+}\right)$and

$$
C_{4} \equiv \operatorname{ess~sup}_{x \in R_{+}}\left(\frac{W_{\alpha}\left[W_{\alpha} v\right]^{p^{\prime}}(x)}{W_{\alpha} v(x)}\right)^{1 / p^{\prime}}<\infty .
$$

Moreover, $\left\|R_{\alpha}\right\| \approx C_{4}$.

Theorem H ([KM2]). Let $1<p<\infty$ and let $0<\alpha<1 / p$. Then the operator $W_{\alpha}$ is bounded from $L^{p}\left(R_{+}\right)$to $L_{v}^{p}\left(R_{+}\right)$if and only if $R_{\alpha} v \in L_{l o c}^{p^{\prime}}\left(R_{+}\right)$and

$$
C_{5} \equiv \underset{x \in R_{+}}{\operatorname{ess} \sup }\left(\frac{R_{\alpha}\left[R_{\alpha} v\right]^{p^{\prime}}(x)}{R_{\alpha} v(x)}\right)^{1 / p^{\prime}}<\infty .
$$

Moreover, $\left\|W_{\alpha}\right\| \approx C_{5}$.

The Volterra-type integral operators with general kernels extending one-sided potentials mainly in the case when $\alpha>1 / p$ were investigated in [M2] (see also [EKM], Section 2.7).

For the applications of the results presented above to the solubility problems of Abel's integral equation and certain superlinear inhomogeneous integral equation we refer to [KM2] (see also [EKM], Sections 2.12, 2.13 and 2.14), where the criteria of the existence of positive solutions of some Volterra-type nonlinear integral equations are obtained. 
2. One-sided potentials with multiple kernels. Now we consider the operator

$$
R_{\alpha, \beta} f(x, y)=\int_{0}^{x} \int_{0}^{y} \frac{f(t, \tau)}{(x-t)^{1-\alpha}(y-\tau)^{1-\beta}} d t d \tau .
$$

When $\alpha=\beta=1$ this operator is the multiple Hardy transform which will be discussed in Section 3.

THEOREM 2.1 ([KM4]). Let $1<p \leq q<\infty, \alpha, \beta>1 / p$. Then the following statements are equivalent:

(i) $R_{\alpha, \beta}$ is bounded from $L^{p}\left(R_{+}^{2}\right)$ to $L_{v}^{q}\left(R_{+}^{2}\right)$;

(ii)

$$
\sup _{a, b>0}\left(\int_{a}^{\infty} \int_{b}^{\infty} v(x, y) x^{(\alpha-1) q} y^{(\beta-1) q} d x d y\right)^{1 / q}(a b)^{1 / p^{\prime}}<\infty
$$

(iii)

$$
\sup _{k, j \in Z}\left(\int_{2^{k}}^{2^{k+1}} \int_{2^{j}}^{2^{j+1}} v(x, y) d x d y\right)^{1 / q} 2^{k(\alpha-1 / p)} 2^{j(\beta-1 / p)}<\infty .
$$

To prove this statement we represent the integral $R_{\alpha, \beta} f$ as follows:

$$
\begin{aligned}
R_{\alpha, \beta} f(x, y)= & \int_{0}^{x / 2} \int_{0}^{y / 2}(\cdots) d t d \tau+\int_{0}^{x / 2} \int_{y / 2}^{y}(\cdots) d t d \tau \\
& +\int_{x / 2}^{x} \int_{0}^{y / 2}(\cdots) d t d \tau+\int_{x / 2}^{x} \int_{y / 2}^{y}(\cdots) d t d \tau \\
\equiv & R_{\alpha, \beta}^{(1)} f(x, y)+R_{\alpha, \beta}^{(2)} f(x, y)+R_{\alpha, \beta}^{(3)} f(x, y)+R_{\alpha, \beta}^{(4)} f(x, y) .
\end{aligned}
$$

Further, the following statement holds.

Lemma 2.1 ([KM4]). Suppose that $1<p \leq q<\infty$. Then the operator $\mathrm{H}_{2}$ is bounded from $L^{p}\left(R_{+}^{2}\right)$ to $L_{u}^{q}\left(R_{+}^{2}\right)$ if and only if

$$
A \equiv \sup _{a, b>0}\left(\left(H_{2}^{\prime} u\right)(a, b)\right)^{1 / q}(a b)^{1 / p^{\prime}}<\infty .
$$

Moreover, $\left\|H_{2}\right\| \approx A$.

This lemma yields

$$
\left\|R_{\alpha, \beta}^{(1)} f\right\|_{L_{v}^{q}\left(R_{+}^{2}\right)} \leq c\|f\|_{L^{p}\left(R_{+}^{2}\right)},
$$

while due to the classical Hardy's inequality (see [HLP], p. 240 for $p=q$ and, e.g., [SKM], Ch. 2, for $p \leq q)$ :

$$
\left(\int_{0}^{\infty}(H f(x))^{q} x^{-q / p^{\prime}-1} d x\right)^{1 / q} \leq c\left(\int_{0}^{\infty}(f(x))^{p} d x\right)^{1 / p}
$$

we have the boundedness of $R^{(i)}, i=1,2$, from $L^{p}\left(R_{+}^{2}\right)$ to $L_{v}^{q}\left(R_{+}^{2}\right)$. Hölder's inequality and simple calculations of integrals guarantee

$$
\left\|R_{\alpha, \beta}^{(4)} f\right\|_{L_{v}^{q}\left(R_{+}^{2}\right)} \leq c\|f\|_{L^{p}\left(R_{+}^{2}\right)} .
$$

This completes the proof of sufficiency. For details see [KM4]. 
It should be mentioned that the condition

$$
\sup _{j}\left(\int_{2^{j}}^{2^{j+1}}\left[\sup _{k}\left(\int_{2^{k}}^{2^{k+1}} v(x, y) d x\right) 2^{k(\alpha p-1)}\right] d y\right) 2^{j(\beta p-1)}<\infty
$$

is sufficient but not necessary for the boundedness of $R_{\alpha, \beta}$ from $L^{p}\left(R_{+}^{2}\right)$ to $L_{v}^{p}\left(R_{+}^{2}\right)$.

EXAMPLE. Let

$$
v(x, y)= \begin{cases}m 2^{-m \alpha p} & \text { if }(x, y) \in\left[2^{m}, 2^{m+1}\right] \times(1,1+1 / m], \\ 2^{-m \alpha p} & \text { for }(x, y) \in\left[2^{m}, 2^{m+1}\right] \times[1+1 / m, 2], \\ x^{-\alpha p} y^{-\beta p} & \text { otherwise }\end{cases}
$$

For such $v$ the condition $(*)$ fails but (i) of Theorem 2.1 holds.

The remaining statements of this section are published in [KM4]-[KM5].

Theorem 2.2. Let $1<p<\infty$. Suppose that $0<\alpha<1 / p$ and $\beta>1 / p$. Then the operator $R_{\alpha, \beta}$ is bounded from $L^{p}\left(R_{+}^{2}\right)$ to $L_{v}^{p}\left(R_{+}^{2}\right)$ if and only if $W_{\alpha} V_{j} \in L_{\text {loc }}^{p^{\prime}}\left(R_{+}\right)$for all $j \in Z$ and

$$
W_{\alpha}\left[W_{\alpha} V_{j}\right]^{p^{\prime}}(x) \leq c W_{\alpha}\left[V_{j}\right](x)
$$

for all $x>0$ and $j \in Z$, where

$$
V_{j}(x) \equiv \int_{2^{j}}^{2^{j+1}} v(x, y) y^{\beta p-1} d y
$$

Theorem 2.3. Let $1<p<q<\infty$. Suppose that $0<\alpha<1 / p$ and $\beta>1 / p$. Then the operator $R_{\alpha, \beta}$ is bounded from $L^{p}\left(R_{+}^{2}\right)$ to $L_{v}^{q}\left(R_{+}^{2}\right)$ if and only if

$$
A \equiv \sup _{\substack{0<h<a \\ j \in Z}}\left(\int_{a}^{a+h} \int_{2^{j}}^{2^{j+1}} v(x, y) d x d y\right)^{1 / q} h^{\alpha-1 / p} 2^{j(\beta-1 / p)}<\infty .
$$

Moreover, $\left\|R_{\alpha, \beta}\right\| \approx A$.

Theorem 2.3 follows from the integral representation

$$
\begin{aligned}
R_{\alpha, \beta} f(x, y) & =\int_{0}^{x} \int_{0}^{y / 2}(\cdots) d t d \tau+\int_{0}^{x} \int_{y / 2}^{y}(\cdots) d t d \tau \\
& \equiv \int_{x / 2}^{x} \int_{0}^{y / 2}(\cdots) d t d \tau+\int_{x / 2}^{x} \int_{y / 2}^{y}(\cdots) d t d \tau
\end{aligned}
$$

inequality (2.1) and Theorem D. Theorem 2.2 can be obtained in a similar manner. In this case we need to use Theorem G instead of Theorem D (see [KM5] for details).

Now we present the conditions which yield the boundedness of truncated and ball potentials with multiple kernels. Let

$$
T_{\alpha, \beta} f(x, y)=\int_{|t| \leq 2|x|} \int_{|\tau| \leq 2|y|} f(t, \tau)|x-t|^{\alpha-n}|y-\tau|^{\beta-n} d t d \tau, \quad \alpha, \beta>0 .
$$

Theorem 2.4. Let $1<p \leq q<\infty$. Suppose that $\alpha, \beta>n / p$. Then the operator $T_{\alpha, \beta}$ is bounded $L^{p}\left(R^{2 n}\right)$ to $L_{v}^{q}\left(R^{2 n}\right)$ if and only if

$$
\sup _{a, b>0}\left(\int_{|x|>a} \int_{|y|>b} v(x, y)|x|^{(\alpha-n) q}|y|^{(\beta-n) q} d x d y\right)^{1 / q}(a b)^{n / p^{\prime}}<\infty .
$$


For the multiple ball potential

$$
B_{\alpha, \beta} f(x, y)=\int_{|t|<|x|} \int_{|\tau|<|y|} f(t, \tau) \frac{\left(|x|^{2}-|t|^{2}\right)^{\alpha}}{|x-t|^{n}} \frac{\left(|y|^{2}-|\tau|^{2}\right)^{\beta}}{|y-\tau|^{n}} d t d \tau, \quad \alpha, \beta>0,
$$

we have

THEOREM 2.5. Let $1<p \leq q<\infty$. Suppose that $\alpha, \beta>n / p$. Then the operator $B_{\alpha, \beta}$ is bounded from $L^{p}\left(R^{2 n}\right)$ to $L_{v}^{q}\left(R^{2 n}\right)$ if and only if

$$
\sup _{a, b>0}\left(\int_{|x|>a} \int_{|y|>b} v(x, y)|x|^{(2 \alpha-n) q}|y|^{(2 \beta-n) q} d x d y\right)^{1 / q}(a b)^{n / p^{\prime}}<\infty .
$$

Now we consider the "mixed potentials" with multiple kernels:

$$
\begin{gathered}
\left(I_{\alpha} R_{\beta}\right) f(x, y)=\int_{0}^{\infty} \int_{0}^{y}|x-t|^{\alpha-1}(y-\tau)^{\beta-1} f(t, \tau) d t d \tau, x, y>0 \\
\left(W_{\alpha} R_{\beta}\right) f(x, y)=\int_{x}^{\infty} \int_{0}^{y}(t-x)^{\alpha-1}(y-\tau)^{\beta-1} f(t, \tau) d t d \tau, \quad x, y>0 .
\end{gathered}
$$

TheOREm 2.6. Let $1<p \leq q<\infty$. Suppose that $0<\alpha<1 / p, \beta>1 / p$. Then the operator $I_{\alpha} R_{\beta}$ is bounded from $L^{p}\left(R_{+}^{2}\right)$ to $L_{v}^{q}\left(R_{+}^{2}\right)$ if and only if

$$
\sup _{\substack{h, a, j \\ 0<h<a, j \in Z}}\left(\int_{a}^{a+h} \int_{2^{j}}^{2^{j+1}} v(x, y) d x d y\right)^{1 / q} h^{\alpha-1 / p} 2^{j(\beta-1 / p)}<\infty .
$$

THEOREM 2.7. Let $1<p<\infty$. Suppose that $0<\alpha<1 / p$ and $\beta>1 / p$. Then the operator $W_{\alpha} R_{\beta}$ is bounded from $L^{p}\left(R_{+}^{2}\right)$ to $L_{v}^{p}\left(R_{+}^{2}\right)$ if and only if $R_{\alpha} V_{j} \in L_{l o c}^{p^{\prime}}\left(R_{+}\right)$for all $j \in Z$ and

$$
R_{\alpha}\left[R_{\alpha} V_{j}\right]^{p^{\prime}}(x) \leq c R_{\alpha}\left[V_{j}\right](x), x>0, j \in Z,
$$

where $V_{j}$ are defined as follows

$$
V_{j}(x) \equiv \int_{2^{j}}^{2^{j+1}} v(x, y) y^{\beta p-1} d y
$$

Theorem 2.8. Let $1<p<\infty$. Suppose that $0<\alpha<1 / p$ and $\beta>1 / p$. Then for the boundedness of $I_{\alpha} R_{\beta}$ from $L^{p}\left(R_{+}^{2}\right)$ to $L_{v}^{p}\left(R_{+}^{2}\right)$, it is necessary and sufficient that $R_{\alpha} V_{j}, W_{\alpha} V_{j} \in L_{l o c}^{p^{\prime}}\left(R_{+}\right)$for all $j \in Z$ and conditions (2.2), (2.4) hold.

To end this section we discuss integral transforms with general multiple kernels.

Let

$$
K f(x, y)=\int_{0}^{x} \int_{0}^{y} f(t, \tau) k_{1}(x, t) k_{2}(y, \tau) d t d \tau, \quad x, y>0,
$$

where $k_{i}(i=1,2)$ are positive kernels.

We say that a kernel $k:\{(x, y): 0<y<x<\infty\} \rightarrow(0, \infty)$ belongs to $V(k \in V)$ if there exists a positive constant $c_{1}$ such that for all $x, t, z$ with $0<t<z<x<\infty$ the inequality

$$
k(x, t) \leq c_{1} k(x, z)
$$


holds, and $k$ belongs to $V_{\lambda}\left(k \in V_{\lambda}\right)(1<\lambda<\infty)$ if there exists a positive constant $c_{2}$ such that for all $x, x>0$, the inequality

$$
\int_{x / 2}^{x} k^{\lambda^{\prime}}(x, y) d y \leq c_{2} x k^{\lambda^{\prime}}(x, x / 2)
$$

is fulfilled, where $\lambda^{\prime}=\lambda /(\lambda-1)$.

For example, if $k(x, y)=(x-y)^{\alpha-1} \chi_{\{(x, y): 0<y<x\}}$ with $1 / p<\alpha \leq 1$, then $k \in V \cap V_{p}$. For other examples of kernels $k$ satisfying the conditions mentioned above see [M2], [EKM], Section 2.7.

Theorem 2.9. Let $1<p \leq q<\infty$. Suppose that the kernels $k_{1}$ and $k_{2}$ belong to $V \cap V_{p}$. Then the following statements are equivalent:

(i) the operator $K$ is bounded from $L^{p}\left(R_{+}^{2}\right)$ to $L_{v}^{q}\left(R_{+}^{2}\right)$;

(ii)

$$
B \equiv \sup _{a, b>0}\left(\int_{a}^{\infty} \int_{b}^{\infty} v(x, y) k_{1}^{q}(x, x / 2) k_{2}^{q}(y, y / 2) d x d y\right)^{1 / q}(a b)^{1 / p^{\prime}}<\infty
$$

(iii)

$$
\tilde{B} \equiv \sup _{k, j \in Z}\left(\int_{2^{k}}^{2^{k+1}} \int_{2^{j}}^{2^{j+1}} v(x, y)(x y)^{q / p^{\prime}} k_{1}^{q}(x, x / 2) k_{2}^{q}(y, y / 2) d x d y\right)^{1 / q}<\infty .
$$

Moreover, $\|K\| \approx B \approx \tilde{B}$.

3. Two-weighted inequalities for two-dimensional Hardy operators. Let us consider the operators

$$
\begin{gathered}
H_{2} f(x, y)=\int_{0}^{x} \int_{0}^{y} f(t, \tau) d t d \tau, \\
H_{2}^{\prime} f(x, y)=\int_{x}^{\infty} \int_{y}^{\infty} f(t, \tau) d t d \tau, \quad x, y>0 .
\end{gathered}
$$

The solution of the two-weight problem for two-dimensional Hardy operator has been given by E. Sawyer [Sa2]:

Theorem I. Let $1<p \leq q<\infty$. Then for the boundedness of the operator $\mathrm{H}_{2}$ from $L_{w}^{p}\left(R_{+}^{2}\right)$ to $L_{v}^{q}\left(R_{+}^{2}\right)$ it is necessary and sufficient that the following three conditions are satisfied:

(i)

$$
\sup _{a, b>0}\left(H_{2}^{\prime} v(a, b)\right)^{1 / q}\left(H_{2} \sigma(a, b)\right)^{1 / p^{\prime}} \equiv A<\infty, \quad \sigma=w^{1-p^{\prime}}
$$

(ii)

$$
\int_{0}^{a} \int_{0}^{b}\left(H_{2} \sigma\right)^{q} v \leq A^{q}\left[H_{2} \sigma(a, b)\right]^{q / p}
$$

for all $a, b>0$;

(iii)

$$
\int_{a}^{\infty} \int_{b}^{\infty}\left(H_{2}^{\prime} v\right)^{p^{\prime}} \sigma \leq A^{p^{\prime}}\left[H_{2}^{\prime} v(a, b)\right]^{p^{\prime} / q^{\prime}}
$$

for all $a, b>0$. 
Earlier in [Sy], [Se] were derived some sufficient conditions for the validity of the corresponding multiple Hardy inequality. When the weight function is a product of two onedimensional weights, i.e. $w(x, y)=w_{1}(x) w_{2}(y)$, the boundedness criteria from $L_{w}^{p}\left(R_{+}^{2}\right)$ to $L_{v}^{q}\left(R_{+}^{2}\right)$ for the operator $H_{2}$ have been found in [W]. Here we present some new results concerning the two-weight inequalities for the operator $\mathrm{H}_{2}$, not necessarily for the weights which are product of two functions of separate variables on the right side.

Definition 3.1. A nonnegative function $\rho: R_{+}^{2} \rightarrow R^{1}$ is said to be a weight function with doubling condition uniformly with respect to $y \in R_{+}$if there exists a positive constant $c$ such that for arbitrary $t>0$ and almost all $x>0$ the condition

$$
\int_{0}^{2 t} \rho(x, y) d y \leq c \int_{0}^{t} \rho(x, y) d y
$$

holds. In this case we write that $\rho \in D C(y)$. Analogously we define the class $D C(x)$.

Let us formulate the results for more general two-dimensional Hardy-type transform

$$
R_{\alpha, \beta} f(x, y)=\int_{0}^{x} \int_{0}^{y} \frac{f(t, \tau)}{(x-t)^{1-\alpha}(y-\tau)^{1-\beta}} d t d \tau, \quad \alpha, \beta \geq 1 .
$$

THEOREM 3.1. Let $1<p \leq q<\infty$ and let $\alpha, \beta \geq 1$. Suppose that $w^{1-p^{\prime}} \in D C(y)$. Then the operator $R_{\alpha, \beta}$ is bounded from $L_{w}^{p}\left(R_{+}^{2}\right)$ to $L_{v}^{q}\left(R_{+}^{2}\right)$ if and only if the following two conditions hold:

$$
\begin{gathered}
A_{1} \equiv \sup _{a, b>0}\left(\int_{0}^{a} \int_{0}^{b} \frac{w^{1-p^{\prime}}(x, y)}{(a-x)^{(1-\alpha) p^{\prime}}} d x d y\right)^{1 / p^{\prime}}\left(\int_{a}^{\infty} \int_{b}^{\infty} \frac{v(x, y)}{y^{(1-\beta) q}} d x d y\right)^{1 / q}<\infty \\
A_{2} \equiv \sup _{a, b>0}\left(\int_{0}^{a} \int_{0}^{b} w^{1-p^{\prime}}(x, y) d x d y\right)^{1 / p^{\prime}}\left(\int_{a}^{\infty} \int_{b}^{\infty} \frac{v(x, y)}{(x-a)^{(1-\alpha) q} y^{(1-\beta) q}} d x d y\right)^{1 / q}<\infty .
\end{gathered}
$$

Moreover, $\left\|R_{\alpha, \beta}\right\| \approx \max \left\{A_{1}, A_{2}\right\}$.

THEOREM 3.2. Let $1<p \leq q<\infty$ and let $\alpha, \beta \geq 1$. Suppose that $w^{1-p^{\prime}} \in D C(x)$. Then the operator $R_{\alpha, \beta}$ is bounded from $L_{w}^{p}\left(R_{+}^{2}\right)$ to $L_{v}^{q}\left(R_{+}\right)$if and only if the following two conditions hold:

$$
\begin{gathered}
B_{1} \equiv \sup _{a, b>0}\left(\int_{0}^{a} \int_{0}^{b} \frac{w^{1-p^{\prime}}(x, y)}{(b-y)^{(1-\beta) p^{\prime}}} d x d y\right)^{1 / p^{\prime}}\left(\int_{a}^{\infty} \int_{b}^{\infty} \frac{v(x, y)}{x^{(1-\alpha) q}} d x d y\right)^{1 / q}<\infty \\
B_{2} \equiv \sup _{a, b>0}\left(\int_{0}^{a} \int_{0}^{b} w^{1-p^{\prime}}(x, y) d x d y\right)^{1 / p^{\prime}}\left(\int_{a}^{\infty} \int_{b}^{\infty} \frac{v(x, y)}{(y-b)^{(1-\beta) q} x^{(1-\alpha) q}} d x d y\right)^{1 / q}<\infty .
\end{gathered}
$$

Moreover, $\left\|R_{\alpha, \beta}\right\| \approx \max \left\{B_{1}, B_{2}\right\}$.

Proofs of Theorems 3.1-3.2 are based on Theorem B and the following statement (see, e.g., [M3]):

Proposition 3.1. Let $1<p \leq q<\infty$ and let $\left\{a_{n}\right\},\left\{b_{n}\right\}$ be positive sequences. The inequality

$$
\left(\sum_{n=-\infty}^{\infty}\left|\sum_{k=-\infty}^{n} g_{k}\right|^{q} a_{n}^{q}\right)^{1 / q} \leq c\left(\sum_{n=-\infty}^{\infty}\left|g_{n}\right|^{p} b_{n}^{p}\right)^{1 / p}
$$


holds with the positive constant $c$ independent of $\left\{g_{k}\right\}\left(g_{k} \in l_{b_{n}^{p}}^{p}(Z)\right)$ if and only if

$$
B=: \sup _{n \in Z}\left(\sum_{k=n}^{\infty} a_{k}^{q}\right)^{1 / q}\left(\sum_{k=-\infty}^{n} b_{k}^{-p^{\prime}}\right)^{1 / p^{\prime}}<\infty .
$$

Moreover, if $c$ is the best constant in (3.2), then

$$
B \leq c \leq B q^{\frac{1}{q}}\left(\frac{q}{q-1}\right)^{(p-1) / p} .
$$

Corollary 3.1. Let $1<p \leq q<\infty$ and let $w^{1-p^{\prime}} \in D C(x)$ or $w^{1-p^{\prime}} \in D C(y)$. Then the operator $\mathrm{H}_{2}$ is bounded from $L_{w}^{p}\left(R_{+}^{2}\right)$ to $L_{v}^{q}\left(R_{+}^{2}\right)$ if and only if condition (3.1) holds.

A more general form of this result is the following statement, which we formulate for $\mathrm{H}_{2}$ :

THEOREM 3.3. Let $1<p \leq q<\infty$. Assume that the weight function $w$ satisfies the condition

$$
\sup _{x>0, k \in Z}\left(\sum_{j=k}^{\infty}\left(\int_{0}^{2^{j}} w^{1-p^{\prime}}(x, y) d y\right)^{1-p}\right)\left(\int_{0}^{2^{k+1}} w^{1-p^{\prime}}(x, y) d x\right)^{p-1}<\infty .
$$

Then the boundedness of $H_{2}$ from $L^{p}\left(R_{+}^{2}\right)$ to $L_{v}^{q}\left(R_{+}^{2}\right)$ is equivalent to the condition (3.1).

The following theorem states that when the weight $w$ on the right-hand side has the form $w(x, y)=w_{1}(x) w_{2}(y)$ then the first condition in Sawyer's theorem is equivalent to the boundedness of the operator $H_{2}$ from $L_{w}^{p}\left(R_{+}^{2}\right)$ to $L_{v}^{q}\left(R_{+}^{2}\right)$.

Theorem 2.4. Let $1<p \leq q<\infty$ and $w(x, y)=w_{1}(x) w_{2}(y)$. Then the operator $H_{2}$ is bounded from $L_{w}^{p}\left(R_{+}^{2}\right)$ to $L_{v}^{q}\left(R_{+}^{2}\right)$ if and only if

$$
A_{1} \equiv \sup _{a, b>0}\left(\int_{0}^{a} \int_{0}^{b} w^{1-p^{\prime}}(x, y) d x d y\right)^{1 / p^{\prime}}\left(\int_{a}^{\infty} \int_{b}^{\infty} v(x, y) d x d y\right)^{1 / q}<\infty .
$$

The results of this section were published in [M3] and announced in [M4].

4. Multiple Riesz potentials. In this section we deal with the following operators:

$$
\begin{gathered}
\left(I_{\alpha, \beta} f\right)(x, y)=\int_{R} \int_{R} f(t, \tau)|x-t|^{\alpha-1}|y-\tau|^{\beta-1} d t d \tau \\
\left(J_{\alpha, \beta} f\right)(x, y)=\int_{R} \int_{|\tau|<2|y|} f(t, \tau)|x-t|^{\alpha-1}|y-\tau|^{\beta-1} d t d \tau,
\end{gathered}
$$

where $0<\alpha, \beta<1$.

Let us first formulate the results for $J_{\alpha, \beta}$.

TheOrem 4.1. Let $1<p<q<\infty, 1 / p-1 / q<\alpha<1,1 / p<\beta<1$. Then the operator $J_{\alpha, \beta}$ is bounded from $L^{p}\left(R^{2}\right)$ to $L_{v}^{q}\left(R^{2}\right)$ if and only if

$$
\sup _{\substack{a \in R \\ r>0 \\ k \in Z}}\left(\int_{a-r}^{a+r} \int_{2^{k}<|y|<2^{k+1}} v(x, y) d x d y\right)^{1 / q} r^{\alpha-1 / p} 2^{k(\beta-1 / p)}<\infty .
$$

In the diagonal case $p=q$ we have 
THEOREM 4.2. Let $1<p<\infty, 0<\alpha<1,1 / p<\beta<1$. Then the operator $J_{\alpha, \beta}$ is bounded from $L^{p}\left(R^{2}\right)$ to $L_{v}^{p}\left(R^{2}\right)$ if and only if there exists a positive constant $c$ such that for a.a. $x \in R$ and $k \in Z$ the inequality

$$
K_{\alpha}\left[K_{\alpha} V_{j}\right]^{p^{\prime}}(x) \leq K_{\alpha}\left[V_{j}\right](x)
$$

holds, where $K_{\alpha}$ is the one-dimensional potential

and

$$
K_{\alpha} f(x)=\int_{R} \frac{f(y)}{|x-y|^{1-\alpha}} d y
$$

$$
V_{j}(x) \equiv \int_{2^{j}<|y|<2^{j+1}} v(x, y)|y|^{\beta p-1} d y .
$$

Further, the following statement holds.

TheOrem 4.3. Let $1<p<q<\infty$. Suppose that $1 / p-1 / q<\alpha<1,1 / p<\beta<1$. Then the two-weight inequality

$$
\left(\int_{R} \int_{R}\left|\left(J_{\alpha, \beta} f\right)(x, y)\right|^{q} v(x, y) d x d y\right)^{1 / q} \leq c\left(\int_{R} \int_{R}|f(x, y)|^{p} u(x) d x d y\right)^{1 / p}
$$

holds if and only if

(i)

$$
\begin{aligned}
& \sup _{\substack{a \in R \\
r>0 \\
k \in Z}}\left(\int_{|x-a|>r} \int_{2^{k}<|y|<2^{k+1}} \frac{v(x, y)}{|x-a|^{(1-\alpha) q}} d x d y\right)^{1 / q} \\
& \quad \times\left(\int_{|x-a|<r} u^{1-p^{\prime}}(x) d x\right)^{1 / p^{\prime}} 2^{k(\beta-1 / p)}<\infty ;
\end{aligned}
$$

(ii)

$$
\begin{aligned}
& \sup _{\substack{a \in R \\
r>0 \\
k \in Z}}\left(\int_{|x-a|<r} \int_{2^{k}<|y|<2^{k+1}} v(x, y) d x d y\right)^{1 / q} \\
& \quad \times\left(\int_{|x-a|>r} \frac{u^{1-p^{\prime}}(x)}{|x-a|^{(\alpha-1) p^{\prime}}} d x\right)^{1 / p^{\prime}} 2^{k(\beta-1 / p)}<\infty .
\end{aligned}
$$

DEFinition 4.1. We say that a two-dimensional weight function $\rho$ defined on $R^{2}$ satisfies the reverse doubling condition at 0 uniformly with respect to $x$ if there exist constants $\eta_{1}>1$ and $\eta_{2}>1$ such that for all $x \in R$ and $r>0$ the inequality

$$
\int_{B\left(0, \eta_{1} r\right)} \rho(x, y) d y \geq \eta_{2} \int_{B(0, r)} \rho(x, y) d y
$$

holds. In this case we write $\rho \in R D_{0}\left(y, \eta_{1}, \eta_{2}\right)$ (or $\left.\rho \in R D_{0}(y)\right)$.

Definition 4.2. The weight $\rho: R^{2} \rightarrow R$ belongs to the class $R D_{\infty}\left(y, \lambda_{1}, \lambda_{2}\right)$ (or $\left.R D_{\infty}(y)\right), \lambda_{1}, \lambda_{2}>1$, if the inequality

$$
\int_{R^{2} \backslash B\left(0, r / \lambda_{1}\right)} \rho(x, y) d y \geq \lambda_{2} \int_{R^{2} \backslash B(0, r)} \rho(x, y) d y
$$

holds for all $r>0$ and $x \in R$. 
Analogously we can define the classes $R D_{0}(x), R D_{\infty}(x)$.

Theorem 4.4. Let $1<p<q<\infty, 0<\alpha, \beta<1$. Suppose that $w^{1-p^{\prime}} \in R D_{0}\left(y, \eta_{1}, \eta_{2}\right)$ and $\frac{w^{1-p^{\prime}}(x, y)}{|y|^{(1-\beta) p^{\prime}}} \in R D_{\infty}\left(y, \mu_{1}, \mu_{2}\right)$. Assume also that $w$ satisfies the condition: there exists a positive constant $c$ such that for all $y \in R$ and $t \in R$ the inequality

$$
\int_{\frac{|y|}{\eta_{1}}<|\tau|<\mu_{1}|y|} \frac{w^{1-p^{\prime}}(t, \tau)}{|y-\tau|^{(1-\beta) p^{\prime}}} d \tau \leq \frac{c}{|y|^{(1-\beta) p^{\prime}}} \int_{\frac{|y|}{\eta_{1} \mu_{1}}<|\tau|<\frac{|y|}{\eta_{1}}} w^{1-p^{\prime}}(t, \tau) d \tau
$$

holds. Then $I_{\alpha, \beta}$ is bounded from $L_{w}^{p}\left(R^{2}\right)$ to $L_{v}^{q}\left(R^{2}\right)$ if and only if the following two conditions are satisfied:

(i)

$$
\begin{aligned}
& \sup _{\substack{a \in R \\
r>0 \\
s>0}}\left(\int_{|x-a|<r} \int_{|y|>s / \mu_{1}^{2}} \frac{w^{1-p^{\prime}}(x, y)}{|y|^{(1-\beta) p^{\prime}}} d x d y\right)^{1 / p^{\prime}} \\
& \quad \times\left(\int_{|x-a|>r} \int_{|y|<s} \frac{v(x, y)}{|x-a|^{(1-\alpha) q}} d x d y\right)^{1 / q}<\infty ;
\end{aligned}
$$

(ii)

$$
\begin{aligned}
& \sup _{\substack{a \in R \\
r>0 \\
s>0}}\left(\int_{|x-a|>r} \int_{|y|>s / \mu_{1}^{2}} \frac{w^{1-p^{\prime}}(x, y)}{\left.|x-a|^{(1-\alpha) p^{\prime}|y|^{(1-\beta) p^{\prime}}} d x d y\right)^{1 / p^{\prime}}}\right. \\
& \quad \times\left(\int_{|x-a|<r} \int_{|y|<s} v(x, y) d x d y\right)^{1 / q}<\infty .
\end{aligned}
$$

REMARK 4.1. For example, the weight function $w(x, y)$ which is increasing with respect to $y$ for a.a. $x \in R$ satisfies the condition (4.1).

Corollary 4.1. Let $1<p<q<\infty, 0<\alpha<1,1 / p<\beta<1$. Then the two-weight inequality

$$
\left(\int_{R} \int_{R}\left|\left(I_{\alpha, \beta} f\right)(x, y)\right|^{q} v(x, y) d x d y\right)^{1 / q} \leq c\left(\int_{R} \int_{R}|f(x, y)|^{p}|y|^{\gamma} d x d y\right)^{1 / p}
$$

holds if and only if

(i)

$$
\beta p-1<\gamma<p-1
$$

(ii)

$$
\sup _{\substack{a \in R \\ r>0 \\ t>0}}\left(\int_{a-r}^{a+r} \int_{|y|>t} \frac{v(x, y)}{|y|^{(1-\alpha) q}} d x d y\right)^{1 / q} r^{\alpha-1 / p} 2^{k\left(-\gamma / p+1 / p^{\prime}\right)}<\infty .
$$

To formulate the next results we shall need some definitions.

Definition 4.3. We say that a weight $u$ defined on $R$ satisfies the reverse Hölder's inequality if there exist $r>1$ and $c>0$ such that

$$
\left(\frac{1}{t} \int_{|x|<t} u^{r}(x) d x\right)^{1 / r} \leq \frac{c}{t} \int_{|x|<t} u(x) d x
$$

holds for all $t>0$. In this case we write $u \in R H_{0}(r)$. 
Note that if the weight function $u$ satisfies condition (4.2), then it also satisfies

$$
\left(\frac{1}{t} \int_{t<|x|<\lambda t} u^{r}(x) d x\right)^{1 / r} \leq \frac{c}{t} \int_{|x|<\lambda t} u(x) d x
$$

for all $t$ and $\lambda>1$, where the constant $c$ is independent of $t$. In the latter case we write $u \in R H_{a n}(r)$.

REMARK 4.2. For example, the power-type weight $u(x)=|x|^{\gamma}$ belongs to $R H_{a n}(r)$ for all $\gamma \in R$.

Definition 4.4. A two-dimensional weight $\rho: R^{2} \rightarrow R$ satisfies the reverse Hölder's inequality uniformly with respect to $x\left(u \in R H_{0}(r, y)\right)$, if there exist $r>1$ and $c>0$ such that for all $t>0$ and $x \in R$ the inequality

$$
\left(\frac{1}{t} \int_{|y|<t} \rho^{r}(x, y) d y\right)^{1 / r} \leq \frac{c}{t} \int_{|y|<t} \rho(x, y) d y
$$

holds. We say that $\rho \in R H_{a n}(r, y)$ if

$$
\left(\frac{1}{t} \int_{t<|y|<\lambda t} \rho^{r}(x, y) d y\right)^{1 / r} \leq \frac{c}{t} \int_{|y|<\lambda t} \rho(x, y) d y
$$

holds, for all $t>0, \lambda>1$ and $x \in R$, and the constant $c$ is independent of $t$ and $x$.

Proposition 4.1. The following statements are equivalent:

(i) The weight $\rho \in R H_{0}(r, y)$;

(ii) $\rho^{r} \in A_{\infty}^{0}(y)$, i.e. there exist positive constants $\delta$ and $c$ such that for all $a>0$ and any measurable subset $E \subset[-a, a]$ the inequality

$$
\frac{\rho_{x}^{r}(E)}{\rho_{x}^{r}([-a, a])} \leq c\left(\frac{|E|}{a}\right)^{\delta}
$$

holds, where $\rho_{x}^{r}(S) \equiv \int_{S} \rho^{r}(x, y) d y$;

(iii) $\rho^{r} \in A_{p}^{0}(y)$ for some $p \geq 1$. By the definition $\rho^{r} \in A_{p}^{0}(y)$ if

$$
\sup _{a, x}\left(\frac{1}{a} \int_{-a}^{a} \rho^{r}(x, y) d y\right)\left(\frac{1}{a} \int_{-a}^{a} \rho^{r /(1-p)}(x, y) d y\right)^{p-1}<\infty \text { for } p>1,
$$

and

$$
\sup _{a, x}\left(\frac{1}{a} \int_{-a}^{a} \rho^{r}(x, y) d y\right) \operatorname{essup}_{y \in[-a, a]} \frac{1}{\rho^{r}(x, y)}<\infty \text { for } p=1 .
$$

The condition (iii) of the latter proposition is the Muckenhoupt's condition written with respect to the intervals of the form $[-a, a]$.

Proposition 4.1 can be obtained in the standard way (see, for example, [Mu2], [GR], Ch. 4, [Fe], [CF], [SW]), where similar problems are discussed for the weight classes $R H$, $A_{\infty}$ and $A_{p}$ which are defined on all cubes of $R^{n}$.

Now we are able to formulate some results concerning the two-weight inequalities for $I_{\alpha, \beta}$.

Let us begin with the case $w \equiv 1$. 
Theorem 4.5. Let $1<p<\infty, 0<\alpha, \beta<1 / p$. Suppose that $v \in R H_{a n}\left(\frac{1}{\alpha p}, x\right)$, $R H_{a n}\left(\frac{1}{\beta p}, y\right)$. Then $I_{\alpha, \beta}$ is bounded from $L^{p}\left(R^{2}\right)$ to $L_{v}^{p}\left(R^{2}\right)$ if and only if

$$
\sup _{\substack{t>0 \\ \tau>0}}\left(\int_{|x|<t} \int_{|y|<\tau} v(x, y) d x d y\right)^{1 / p} t^{\alpha-1 / p} \tau^{\beta-1 / p}<\infty .
$$

In the case of two weights we have

THEOREM 4.6. Let $1<p<\infty, 0<\alpha, \beta<1$. Suppose that the weights $v$ and $w^{1-p^{\prime}}$ belong to the classes $R H_{0}\left(\frac{1}{\alpha}, x\right) ; R H_{0}\left(\frac{1}{\beta}, y\right)$. Then $I_{\alpha, \beta}$ is bounded from $L_{w}^{p}\left(R^{2}\right)$ to $L_{v}^{p}\left(R^{2}\right)$ if and only if

$$
\begin{aligned}
B \equiv \sup _{\substack{t>0 \\
\tau>0}} t^{\alpha-1} \tau^{\beta-1}\left(\int_{|x|<t} \int_{|y|<\tau} v(x, y) d x d y\right)^{1 / p} & \\
& \times\left(\int_{|x|<t} \int_{|y|<\tau} w^{1-p^{\prime}}(x, y) d x d y\right)^{1 / p^{\prime}}<\infty .
\end{aligned}
$$

Remark 4.3. According to Proposition 4.1, Theorem 4.6 remains true if we replace the assumption $v, w^{1-p^{\prime}} \in R H_{0}\left(\frac{1}{\alpha}, x\right), R H_{0}\left(\frac{1}{\beta}, y\right)$ by the condition $v^{1 / \alpha}, w^{\frac{1-p^{\prime}}{\alpha}} \in A_{\infty}^{0}(x)$; $v^{1 / \beta}, w^{\frac{1-p^{\prime}}{\beta}} \in A_{\infty}^{0}(y)$.

Theorem 4.7. Let $1<p<\infty, 0<\alpha, \beta<1$. Suppose that $v$ and $w^{1-p^{\prime}}$ belong to the classes $R D_{0}(x), R D_{0}(y), R H_{a n}(1 / \alpha, x), R H_{a n}(1 / \beta, y)$. Then $I_{\alpha, \beta}$ is bounded from $L_{w}^{p}\left(R^{2}\right)$ to $L_{v}^{p}\left(R^{2}\right)$ if and only if (4.4) holds.

Now we give sketches of the proofs of the main results of this section.

Theorems 4.1, 4.3 and 4.4 follow from the following lemmas:

Lemma 4.1. Let $1<p<q<\infty, 0<\alpha, \beta<1$. Suppose that $w^{1-p^{\prime}} \in R D_{0}\left(y, \eta_{1}, \eta_{2}\right)$. Then the operator

$$
I_{\alpha, \beta}^{(1)} f(x, y)=\int_{R} \int_{|\tau|<|y| / \eta_{1}} f(t, \tau)|x-t|^{\alpha-1}|y-\tau|^{\beta-1} d t d \tau
$$

is bounded from $L_{w}^{p}\left(R^{2}\right)$ to $L_{v}^{q}\left(R^{2}\right)$ if and only if

(a)

$$
\begin{aligned}
& \sup _{\substack{a \in R \\
r>0 \\
s>0}}\left(\int_{|x-a|<r} \int_{|y|<s} w^{1-p^{\prime}}(x, y) d x d y\right)^{1 / p^{\prime}} \\
& \quad \times\left(\int_{|x-a|>r} \int_{|y|>s} \frac{v(x, y)}{|x-a|^{(1-\alpha) q}|y|^{(1-\beta) q}} d x d y\right)^{1 / q}<\infty ;
\end{aligned}
$$

(b)

$$
\begin{aligned}
& \sup _{\substack{a \in R \\
r>0 \\
s>0}}\left(\int_{|x-a|>r} \int_{|y|<s} \frac{w^{1-p^{\prime}}(x, y)}{|x-a|^{(1-\alpha) p^{\prime}}} d x d y\right)^{1 / p^{\prime}} \\
& \qquad \times\left(\int_{|x-a|<r} \int_{|y|>s} \frac{v(x, y)}{|y|^{(1-\beta) q}} d x d y\right)^{1 / q}<\infty .
\end{aligned}
$$


Lemma 4.2. Let $1<p<q<\infty, 0<\alpha, \beta<1$. Suppose that $w^{1-p^{\prime}} \in R D_{\infty}\left(y, \mu_{1}, \mu_{2}\right)$. Then the operator

$$
I_{\alpha, \beta}^{(2)} f(x, y)=\int_{R} \int_{|\tau|>\mu_{1}|y|} f(t, \tau)|x-t|^{\alpha-1}|y-\tau|^{\beta-1} d t d \tau
$$

is bounded from $L_{w}^{p}\left(R^{2}\right)$ to $L_{v}^{p}\left(R^{2}\right)$ if and only if $(i)$ and (ii) of Theorem 4.4 hold.

These lemmas can be proved using Theorem A of Section 1 and two-weight criteria for the discrete Hardy operator (see Proposition 3.1). Further, due to the condition $w^{1-p^{\prime}} \in$ $R D_{0}(y)$ we have that (i) and (ii) of Theorem 4.4 guarantees (a) and (b) of Lemma 4.1.

The next lemma follows from Theorem A, Hölder's inequalities and Proposition 3.1.

Lemma 4.3. Let $1<p<q<\infty, 0<\alpha, \beta<1$. Suppose that $w^{1-p^{\prime}}$ satisfies condition (4.1). Then conditions $(i)$ and (ii) of Theorem 4.4 guarantees the boundedness of the operator

$$
I_{\alpha, \beta}^{(3)} f(x, y)=\int_{R} \int_{|y| / \eta_{1}<|\tau|<\mu_{1}|y|} f(t, \tau)|x-t|^{\alpha-1}|y-\tau|^{\beta-1} d t d \tau
$$

from $L_{w}^{p}\left(R^{2}\right)$ to $L_{v}^{p}\left(R^{2}\right)$.

Now representing $I_{\alpha, \beta}$ as

$$
I_{\alpha, \beta} f=I_{\alpha, \beta}^{(1)} f+I_{\alpha, \beta}^{(2)} f+I_{\alpha, \beta}^{(3)}
$$

and using Lemmas 4.1-4.3 we get sufficiency of Theorem 4.4. Necessity follows easily applying the two-weight inequality to the appropriate class of functions. Theorem 4.1 and 4.3 can be derived from Theorem 4.4. In this case $\mu_{1}, \mu_{2}, \eta_{1}, \eta_{2}=2$ and the conditions $w^{1-p^{\prime}} \in R D_{0}(y) w^{1-p^{\prime}}(x, y) /|y|^{(1-\beta) p^{\prime}} \in R D_{\infty}(y)$ are automatically satisfied.

The proof of Theorem 4.2 is based on the appropriate result concerning the onedimensional potential $K_{\alpha}$.

To prove Theorems 4.5 and 4.7 we need the following statements:

Proposition 4.2. Let $1<p<\infty, 0<\alpha<1 / p$. Suppose that a one-dimensional weight $u$ belong to $R H_{a n}\left(\frac{1}{\alpha p}\right)$. Then $K_{\alpha}$ is bounded from $L^{p}(R)$ to $L_{v}^{p}(R)$ if and only if

$$
\sup _{t>0} t^{\alpha p-1} \int_{|x|<t} v(x) d x<\infty .
$$

Proposition 4.3. Let $1<p<\infty, 0<\alpha<1$. Suppose that one-dimensional weights $u_{1}^{1-p^{\prime}}$ and $u_{2}$ belong to $R H_{a n}\left(\frac{1}{\alpha}\right)$. Then $K_{\alpha}$ is bounded from $L_{u_{1}}^{p}(R)$ to $L_{u_{2}}^{p}(R)$ if and only if the following three simple conditions are satisfied:

$(i)$

$$
\sup _{t>0} t^{\alpha-1}\left(\int_{-t}^{t} u_{2}(x) d x\right)^{1 / p}\left(\int_{-t}^{t} u_{1}^{1-p^{\prime}}(x) d x\right)^{1 / p^{\prime}}<\infty
$$

(ii)

$$
\sup _{t>0}\left(\int_{|x|>t} u_{2}(x)|x|^{(\alpha-1) p} d x\right)^{1 / p}\left(\int_{|x|<t} u_{1}^{1-p^{\prime}}(x) d x\right)^{1 / p^{\prime}}<\infty ;
$$


(iii)

$$
\sup _{t>0}\left(\int_{|x|<t} u_{2}(x) d x\right)^{1 / p}\left(\int_{|x|>t} u_{1}^{1-p^{\prime}}(x)|x|^{(\alpha-1) p^{\prime}} d x\right)^{1 / p^{\prime}}<\infty .
$$

It should be noted that when the weights $u_{1}^{1-p^{\prime}}$ and $u_{2}$ belong to $R H_{0}(1 / \alpha)$, then they satisfy doubling condition for all balls of the type $B(0, r)$, and consequently we have $(i) \Leftrightarrow(i i) \Leftrightarrow(i i i)$. So we can formulate

Proposition 4.4. Let $1<p<\infty, 0<\alpha<1$. Suppose that one-dimensional weights $u_{1}^{1-p^{\prime}}$ and $u_{2}$ belong to $R H_{0}\left(\frac{1}{\alpha}\right)$. Then $K_{\alpha}$ is bounded from $L_{u_{1}}^{p}(R)$ to $L_{u_{2}}^{p}(R)$ if and only if (4.5) holds.

REMARK 4.4. This statement can be also formulated for the Riesz potential $I_{\alpha}$ defined on $R^{n}$. Namely if the weights $u_{1}^{1-p^{\prime}}$ and $u_{2}$ defined on $R^{n}$ satisfy the reverse Hölder's inequality for all balls centered at the origin and for the parameter $n / \alpha$ (or if $u_{1}^{n / \alpha(1-p)}$, $u_{2}^{n / \alpha}$ belongs to the class $A_{\infty}$ defined not over all balls but on the balls centered at the origin), then $I_{\alpha}$ is bounded from $L_{u_{1}}^{p}$ to $L_{u_{2}}^{p}$ if and only if

$$
\sup _{t>0} t^{\alpha / n-1}\left(\int_{B(0, t)} u_{2}(x) d x\right)^{1 / p}\left(\int_{B(0, t)} u_{1}^{1-p^{\prime}}(x) d x\right)^{1 / p^{\prime}}<\infty .
$$

On the other hand, in the paper $[\mathrm{P}]$ it is shown that for the boundedness of $I_{\alpha}$ from $L_{u_{1}}^{p}\left(R^{n}\right)$ to $L_{u_{2}}^{p}\left(R^{n}\right)$ it is necessary and sufficient that

$$
\sup _{Q}|Q|^{\alpha / n-1}\left(\int_{Q} u_{2}(x) d x\right)^{1 / p}\left(\int_{Q} u_{1}^{1-p^{\prime}}(x) d x\right)^{1 / p^{\prime}}<\infty,
$$

where the supremum is taken over all dyadic cubes, provided that $u_{1}^{1-p^{\prime}}$ and $u_{2}$ belong to the class $A_{\infty}$ defined with respect to all dyadic cubes in $R^{n}$.

Propositions 4.3 and 4.4 can be obtained using two-weight criteria for the Hardy-type operators

$$
H f(x)=\int_{|y|<|x|} f(y) d y ; \quad H^{\prime} f(x)=\int_{|y|>|x|} f(y) d y
$$

and the Hardy-Littlewood-Sobolev theorem for the operator $K_{\alpha}$.

From Proposition 4.2 it follows

Lemma 4.4. Let $1<p<\infty, 0<\alpha, \beta<1 / p$. Assume that $v \in R H_{a n}\left(\frac{1}{\alpha p}, x\right)$. Then the following are equivalent:

(i) The operator

$$
J_{\alpha, \beta}^{(1)} f(x, y)=\int_{R} \int_{|\tau|<|y| / 2} f(t, \tau)|x-t|^{\alpha-1}|y-\tau|^{\beta-1} d t d \tau
$$

is bounded from $L^{p}\left(R^{2}\right)$ to $L_{v}^{p}\left(R^{2}\right)$;

(ii) The operator

$$
J_{\alpha, \beta}^{(2)} f(x, y)=\int_{R} \int_{|\tau|>2|y|} f(t, \tau)|x-t|^{\alpha-1}|y-\tau|^{\beta-1} d t d \tau
$$

is bounded from $L^{p}\left(R^{2}\right)$ to $L_{v}^{p}\left(R^{2}\right)$; 
(iii) Condition (4.3) holds.

Proof of Theorem 4.5. Let us represent $I_{\alpha, \beta}$ as follows:

$$
I_{\alpha, \beta} f=\sum_{i=1}^{3} J_{\alpha, \beta}^{(i)} f
$$

where $J^{(1)}$ and $J^{(2)}$ are defined in the latter lemma and

$$
J_{\alpha, \beta}^{(3)} f(x, y) \equiv \int_{R} \int_{|y| / 2<|\tau|<2|y|} f(t, \tau)|x-t|^{\alpha-1}|y-\tau|^{\beta-1} d t d \tau \text {. }
$$

Lemma 4.4 guarantees the boundedness of $J_{\alpha, \beta}^{(1)}$ and $J_{\alpha, \beta}^{(2)}$. Further, the following estimate is obvious:

$$
\begin{aligned}
J_{\alpha, \beta}^{(3)} f & \leq \int_{|t|<|x| / 2} \int_{R}(\cdots)+\int_{|t|>|x| / 2} \int_{R}(\cdots)+\int_{|x| / 2<|t|<2|x|} \int_{|y| / 2<|\tau|<2|y|}(\cdots) \\
& \equiv \sum_{j=1}^{3} J_{\alpha, \beta}^{(3, j)} f .
\end{aligned}
$$

The operators $J_{\alpha, \beta}^{(3,1)}$ and $J_{\alpha, \beta}^{(3,2)}$ are similar to $J_{\alpha, \beta}^{(1)}$ and $J_{\alpha, \beta}^{(2)}$ respectively. If we replace the assumption $v \in R H_{a n}\left(\frac{1}{\alpha p}, x\right)$ by $v \in R H_{a n}\left(\frac{1}{\beta p}, y\right)$, then the statement analogous to Lemma 4.4 holds for $J_{\alpha, \beta}^{(3,1)}$ and $J_{\alpha, \beta}^{(3,2)}$. Further, condition (4.3) guarantees also the boundedness of $J_{\alpha, \beta}^{(3,3)}$ under the restriction $v \in R H_{a n}\left(\frac{1}{\alpha p}, x\right), v \in R H_{a n}\left(\frac{1}{\beta p}, y\right)$.

The proof of Theorem 4.7 is similar to that of Theorem 4.5. In this case we use Proposition 4.3 and the reverse doubling condition. The Hardy-Littlewood-Sobolev and generalized Minkowski's inequalities yield the norm estimate for $J_{\alpha, \beta}^{(3,3)}$.

To get Theorem 4.6 we need

LEMMA 4.5. Let a weight $\rho(x, y)$ belongs to $R H_{0}(r, y)$ for some $r>1$. Then $\rho \in D C_{0}(y)$, i.e. there exists a positive constant $b$ such that for all $t>0$ and $x \in R$ the inequality

$$
\int_{|x|<2 t} \rho(x, y) d y \leq b \int_{|x|<t} \rho(x, y) d y
$$

holds.

Further, it is known (see, e.g., [ST]) that $D C_{0}(y) \Rightarrow R D_{0}(y)$. Now Theorem 4.7 completes the proof of Theorem 4.6 .

5. Weighted inequalities for fractional integrals on nonhomogeneous spaces. Throughout this section we assume that $(X, \mathbf{d}, \mu)$ is a topological space $X$, endowed with a complete measure $\mu$ such that the space of compactly supported continuous functions is dense in $L^{1}(X, \mu)$ and there exists a non-negative real-valued function (quasimetric) d : $X \times X \rightarrow R^{1}$ satisfying the conditions:

(i) $\mathbf{d}(x, x)=0$ for arbitrary $x \in X$;

(ii) $\mathbf{d}(x, y)>0$ for arbitrary $x, y \in X, x \neq y$; 
(iii) there exists a positive constant $a_{0}$ such that for all $x, y \in X$ the following inequality holds:

$$
\mathbf{d}(x, y) \leq a_{0} \mathbf{d}(y, x)
$$

(iv) there exists a positive constant $a_{1}$ such that the inequality

$$
\mathbf{d}(x, y) \leq a_{1}(\mathbf{d}(x, z)+\mathbf{d}(z, y))
$$

holds for arbitrary $x, y, z \in X$;

(v) for every neighbourhood $V$ of the point $x \in X$ there exists a positive number $r$ such that the ball

$$
B(x, r)=\{y \in X: \mathbf{d}(x, y)<r\}
$$

with center in $x$ and radius $r$ is contained in $V$;

(vi) the balls $B(x, r)$ are measurable for all $x \in X, r>0$ and, in addition, $0<$ $\mu B(x, r)<\infty$.

The spaces $(X, \mathbf{d}, \mu)$ with the above mentioned properties are called nonhomogeneous spaces.

In the sequel we shall assume that $\mu(X)=\infty, \mu\{a\}=0$ for all $a \in X$; and $B\left(x, r_{2}\right) \backslash$ $B\left(x, r_{1}\right) \neq \emptyset$ for all $x, r_{1}$ and $r_{2}\left(x \in X, 0<r_{1}<r_{2}<\infty\right)$.

We consider the integral operator of the form:

$$
T_{\alpha} f(x)=\int_{X} \frac{f(y)}{\mathbf{d}(x, y)^{s-\alpha}} d \mu(y), \quad 0<\alpha<s .
$$

Theorem 5.1 ([KM3]). Let $1<p<q<\infty$. The operator $T_{\alpha}$ is bounded from $L^{p}(X)$ into $L^{q}(X)$ if and only if there exists a positive constant $c>0$ such that

$$
\mu B(x, r) \leq c r^{\beta}, \beta=\frac{p q(s-\alpha)}{p q+p-q},
$$

for arbitrary balls $B(x, r)$.

The latter result in the case of Euclidean spaces has been obtained by the first author in $[\mathrm{K} 1]-[\mathrm{K} 2]$.

In particular, from the latter statement it follows

Corollary 5.1. Let $0<\alpha<s, 1<p<s / \alpha$ and $1 / q=1 / p-\alpha / s$. Then $T_{\alpha}$ acts boundedly from $L^{p}(X)$ into $L^{q}(X)$ if and only if

$$
\mu B(x, r) \leq c r^{s} .
$$

Sufficiency of this corollary in the case when $\mathbf{d}$ is a metric has been independently considered in [GG] (for the sufficient condition in the case of $X=R^{n}$ see also [GM]).

Now we concentrate on weighted inequalities for $T_{\alpha}$, in particular we have

Theorem 5.2 ([KM6]). Let $s$ be a positive number. Suppose that $1<p \leq q<\infty$, $s / p-s / q \leq \alpha<s, \alpha \neq s / p, \alpha p-s<\beta<s(p-1)$ and $\lambda=q(s / p+\beta / p-\alpha)-s$. Then the inequality

$$
\left(\int_{X}\left|T_{\alpha} f(x)\right|^{q} \mathbf{d}\left(x_{0}, x\right)^{\lambda} d \mu(x)\right)^{1 / q} \leq c\left(\int_{X}|f(x)|^{p} \mathbf{d}\left(x_{0}, x\right)^{\beta} d \mu(x)\right)^{1 / p}
$$


for the operator $T_{\alpha}$ with the positive constant $c$ independent of $f$ and $x_{0}$ holds if and only if

$$
\sup _{a \in X, r>0} \frac{\mu B(a, r)}{r^{s}}<\infty .
$$

The proof of this statement is based on Corollary 5.1 and the following theorems concerning the Hardy-type operators defined on measure spaces:

$$
\begin{aligned}
& H_{x_{0}} f(x)=\int_{\left\{y: \mathbf{d}\left(x_{0}, y\right) \leq \mathbf{d}\left(x_{0}, x\right)\right\}} f(y) d \mu(y), \\
& H_{x_{0}}^{\prime} f(x)=\int_{\left\{y: \mathbf{d}\left(x_{0}, y\right) \geq \mathbf{d}\left(x_{0}, x\right)\right\}} f(y) d \mu(y),
\end{aligned}
$$

where $x_{0}$ is a fixed point of $X$.

Theorem C ([EKM], Ch. 1). Let $1<p \leq q<\infty$. Suppose that $v$ and $w$ are $\mu$ - a.e. positive functions on $X$. Then

(a) The operator $H_{x_{0}}$ is bounded from $L_{w}^{p}(X)$ to $L_{v}^{q}(X)$ if and only if

$$
A_{1} \equiv \sup _{t \geq 0}\left(\int_{\left\{y: \mathbf{d}\left(x_{0}, y\right) \geq t\right\}} v(y) d \mu(y)\right)^{1 / q}\left(\int_{\left\{y: \mathbf{d}\left(x_{0}, y\right) \leq t\right\}} w^{1-p^{\prime}}(y) d \mu(y)\right)^{1 / p^{\prime}}<\infty,
$$

$p^{\prime}=p /(p-1)$;

(b) The operator $H_{x_{0}}^{\prime}$ is bounded from $L_{w}^{p}(X)$ to $L_{v}^{q}(X)$ if and only if

$$
A_{2} \equiv \sup _{t \geq 0}\left(\int_{\left\{y: \mathbf{d}\left(x_{0}, y\right) \leq t\right\}} v(y) d \mu(y)\right)^{1 / q}\left(\int_{\left\{y: \mathbf{d}\left(x_{0}, y\right) \geq t\right\}} w^{1-p^{\prime}}(y) d \mu(y)\right)^{1 / p^{\prime}}<\infty .
$$

Moreover, there exist positive constants $c_{j}, j=1, \ldots, 4$, depending only on $p$ and $q$ such that

$$
c_{1} A_{1} \leq\left\|H_{x_{0}}\right\| \leq c_{2} A_{1}, \quad c_{3} A_{2} \leq\left\|H_{x_{0}}^{\prime}\right\| \leq c_{4} A_{2} .
$$

REMARK 5.1. It follows immediately from (5.2) that $\mu\{a\}=0$ for all $a \in X$. Therefore in sufficiency of Theorem 5.2 we do not require that the measure $\mu$ has any atoms.

From Theorem 5.2 it is easy to obtain the next corollary for the operator

$$
I_{\alpha}^{x_{0}} f(x)=\mathbf{d}\left(x_{0}, x\right)^{-\alpha s} \int_{X} \frac{f(y)}{\mathbf{d}(x, y)^{s-\alpha}} d \mu(y) .
$$

Corollary 5.2. Let $1<p<\infty, 0<\alpha<s / p$. Then the inequality

$$
\left(\int_{X}\left|I_{\alpha}^{x_{0}} f(x)\right|^{p} d \mu(x)\right)^{1 / p} \leq c\left(\int_{X}|f(x)|^{p} d \mu(x)\right)^{1 / p},
$$

where the positive constant $c$ does not depend on $x_{0}$ and $f$, holds if and only if the measure $\mu$ satisfies the condition (5.2).

It should be stressed that Theorem 5.2 contains the extension of the well-known theorem by E. M. Stein and G. Weiss [StW] concerning two-weight inequality for the Riesz potentials.

A non-negative Borel measure $m$ on $\mathbb{C}$ is called a Radon measure if $m$ is finite on compact sets and

$$
m(A)=\sup m(K)=\inf m(U)
$$


for every Borel set $A$, where the supremum is taken over all compact sets $K \subset A$ and the infimum is over all open sets $U$ containing $A$. We say that a Borel measure $m$ on $\mathbb{C}$ is a Carleson measure if $m$ is a Radon measure and there exists a constant $C:=C(m) \geq 0$ such that

$$
m(D(z, \varepsilon)) \leq C \varepsilon
$$

for all disks $D(z, \varepsilon):=\{\tau \in \mathbb{C}:|\tau-z|<\varepsilon\}$.

Proposition 5.1. Let $m$ be a Radon measure on $\mathbb{C}$. Suppose that $1<p \leq q<\infty$, $1 / p-1 / q \leq \alpha<1, \alpha \neq 1 / p, \alpha p-1<\beta<p-1$ and $\lambda=q(1 / p+\beta / p-\alpha)-1$. Then the two-weight inequality

$$
\left(\int_{\mathbb{C}}\left|K_{\alpha} f(z)\right|^{q}\left|z-z_{0}\right|^{\lambda} d m(z)\right)^{1 / q} \leq c\left(\int_{\mathbb{C}}|f(z)|^{p}\left|z-z_{0}\right|^{\beta} d m(z)\right)^{1 / p}
$$

for the operator

$$
K_{\alpha} f(z)=\int_{\mathbb{C}} \frac{f(\zeta)}{|\zeta-z|^{1-\alpha}} d m(\zeta)
$$

with the positive constant $c$ independent of $f$ and $z_{0}, z_{0} \in \mathbb{C}$, holds if and only if $m$ is a Carleson measure.

\section{References}

[AS] K. F. Andersen and E. T. Sawyer, Weighted norm inequalities for the RiemannLiouville and Weyl fractional operators, Trans. Amer. Math. Soc. 308 (1988), 547558.

[B1] O. V. Besov, Embeddings of differential function spaces with variable smoothness, Trudy Mat. Inst. Steklov. 214 (1997), 25-58 (in Russian).

[B2] O. V. Besov, Weighted spaces of differentiable functions with variable smoothness, Trudy Mat. Inst. Steklov. 210 (1995), 31-40 (in Russian).

[B3] O. V. Besov, On compact embedding of weighted Sobolev spaces on the domain with irregular boundaries, Trudy Mat. Inst. Steklov. 231 (2001), 72-93 (in Russian).

[BK] A. Böttcher and Y. I. Karlovich, Carleson Curves, Muckenhoupt Weights, and Toeplitz Operators, Birkhäuser Verlag, Basel, 1997.

[CF] R. Coifman and C. Fefferman, Weighted norm inequalities inequalities for maximal functions and singular integrals, Studia Math. 51 (1974), 241-250.

[EKM] D. E. Edmunds, V. Kokilashvili and A. Meskhi, Bounded and Compact Integral Operators, Kluwer Academic Publishers, Dordrecht, Boston, London, 2002.

[Fe] R. Fefferman, Multiparameter Fourier analysis, in: Beijing Lectures in Harmonic Analysis (Beijing, 1984), Ann. of Math. Stud. 112, Princeton Univ. Press, Princeton, NJ, 1986, 47-130.

[Ga] M. Gabidzashvili, Weighted inequalities for anisotropic potentials, Trudy Tbiliss. Mat. Inst. Razmadze 82 (1986), 25-36 (in Russian).

[GK] M. Gabidzashvili and V. Kokilashvili, Two-weight weak type inequalities for fractional type integrals, preprint No. 45, Mathematical Institute Czech Acad. Sci., Prague, 1989. 
[GG] J. García-Cuerva and A. E. Gatto, Boundedness properties of fractional integral operators associated to non-doubling measures, Studia Math. 162 (2004), 245-261.

[GM] J. García-Cuerva and J. M. Martell, Two-weight norm inequalities for maximal operators and fractional integrals on nonhomogeneous spaces, Indiana Univ. Math. J. 50 (2001), , 1241-1280.

[GR] J. García-Cuerva and J. L. Rubio de Francia, Weighted Norm Inequalities and Related Topics, North-Holland, Amsterdam, 1985.

[GGKK] I. Genebashvili, A. Gogatishvili, V. Kokilashvili and M. Krbec, Weight Theory for Integral Transforms on Spaces of Homogeneous Type, Pitman Monographs and Surveys in Pure and Applied Mathematics 92, Longman, Harlow, 1998.

[K1] V. M. Kokilashvili, On Hardy's inequalities in weighted spaces, Soobshch. Akad. Nauk Gruz. SSR 96 (1979), 37-40 (in Russian).

[K2] V. Kokilashvili, Maximal functions and singular integrals in weighted function spaces, Trudy Mat. Inst. Razmadze 25 (1985), 1-114 (in Russian).

[K3] V. Kokilashvili, Weighted estimates for classical integral operators, in: Nonlinear Analysis, Function Spaces and Appl. IV, Teubner-Verlag, Leipzig, 1990, 86-103.

[K4] V. Kokilashvili, Potential on thin sets, in: Function Spaces and Appl. Diff. Equat., Izd. Univ. Druzhby Narodov, Moskva, 1992, 25-47 (in Russian).

[KK] V. Kokilashvili and M. Krbec, Weighted Inequalities in Lorentz and Orlicz Spaces, World Scientific, Singapore, 1991.

[KM1] V. Kokilashvili and A. Meskhi, Boundedness and compactness criteria for some classical integral operators, in: Function Spaces V (Poznań, 1998) H. Hudzik and L. Skrzypczak (eds.), Lecture Notes in Pure and Applied Mathematics, 213, Marcel Dekker, New York, 2000, 279-296.

[KM2] V. Kokilashvili and A. Meskhi, On a trace inequality for one-sided potentials and applications to the solvability of nonlinear integral equations, Georgian Math. J. 18 (2001), 521-536.

[KM3] V. Kokilashvili and A. Meskhi, Fractional integrals on measure spaces, Fract. Calc. Appl. Anal. 4 (2001), 1-24.

[KM4] V. Kokilashvili and A. Meskhi, On one-sided potentials with multiple kernels, Integral Trans. Spec. Functions 16 (2005), 669-683.

[KM5] V. Kokilashvili and A. Meskhi, On a trace inequality for one-sided potentials with multiple kernels, Fract. Calc. Appl. Anal. 6 (2003), 461-472.

[KM6] V. Kokilashvili and A. Meskhi, On some weighted inequalities for fractional integrals defined on nonhomogeneous spaces, Z. Anal. Anwend. 24 (2005), 871-885.

[KP] A. Kufner and L. E. Persson, Weighted Inequalities of Hardy Type, World Scientific, Singapore, 2003.

[MS] F. J. Martin-Reyes and E. Sawyer, Weighted inequalities for Riemann-Liouville fractional integrals of order one and greater, Proc. Amer. Math. Soc. 106 (1989), 727-733.

[Ma] V. G. Maz'ya, Sobolev Spaces, Springer, Berlin, 1985.

[MV] V. G. Maz'ya and I. E. Verbitsky, Capacitary inequalities for fractional integrals, with applications to partial differential equations and Sobolev multipliers, Ark. Mat. 33 (1995), 81-115.

[M1] A. Meskhi, Solution of some weight problems for the Riemann-Liouville and Weyl operators, Georgian Math. J. 5 (1998), 565-574.

[M2] A. Meskhi, Criteria for the boundedness and compactness of integral transforms with positive kernels, Proc. Edinburgh Math. Soc. 44 (2001), 267-284. 
[M3] A. Meskhi, A note on two-weight inequalities for multiple Hardy-type operators, J. Function Spaces Appl. 3 (2005), 223-237.

[M4] A. Meskhi, Two-weight criteria for the two-dimensional Hardy-type operators, Proc. A. Razmadze Math. Inst. 136 (2004).

[Mu1] B. Muckenhoupt, Hardy's inequality with weights, Studia Math. 44 (1972), 31-38.

[Mu2] B. Muckenhoupt, Weighted norm inequalities for the Hardy maximal function, Trans. Amer. Math. Soc. 165 (1972), 207-274.

[NS] J. Newman and M. Solomyak, Two-sided estimates on singular values for a class of integral operators on the semi-axis, Integral Equations Operator Theory 20 (1994), 335-349.

[OK] B. Opic and A. Kufner, Hardy-Type Inequalities, Pitman Research Notes in Math. Series 219, Longman Sci. and Tech., Harlow, 1990.

[P] C. Perez, Two-weight norm inequalities for Riesz potentials and uniform $L^{p}$-weighted Sobolev inequalities, Indiana Univ. Math. J. 39 (1990), 31-44.

[Pr] D. V. Prokhorov, On the boundedness of a class of integral operators, J. London Math. Soc. 61 (2000), 617-628.

[PS] D. V. Prokhorov and V. Stepanov, Weighted estimates for the Riemann-Liouville operators and applications, Trudy Mat. Inst. Steklov. 243 (2003), 289-312 (in Russian).

[SKM] S. Samko, A. A. Kilbas and O. I. Marichev, Fractional Integrals and Derivatives. Theory and Applications, Gordon and Breach, London, 1993.

[Sa1] E. T. Sawyer, A two-weight weak type inequality for fractional integrals, Trans. Amer. Math. Soc. 281 (1984), 339-345.

[Sa2] E. Sawyer, Weighted inequalities for the two-dimensional Hardy operator, Studia Math. 82 (1985), 1-11.

[SWZ] E. T. Sawyer, R. L. Wheeden and S. Zhao, Weighted norm inequalities for operators of potential type and fractional maximal functions, Potential Anal. 5 (1996), 523-580.

[Se] V. N. Sedov, Weighted spaces. The imbedding theorem, Differentsial'nye Uravneniya 8 (1972), 1452-1462 (in Russian).

[StW] E. M. Stein and G. Weiss, Fractional integrals on n-dimensional Euclidean spaces, J. Math. Mech. 7 (1958), 503-514.

[St1] V. D. Stepanov, Weighted inequalities of Hardy type for higher derivatives, and their applications, Soviet Math. Dokl. 38 (1989), 389-393.

[St2] V. D. Stepanov, Weighted norm inequalities for integral operators and related topics, in: Nonlinear Analysis, Function Spaces and Applications, 5, Olimpia Press, Prague, 1994, 139-176.

[ST] J. O. Strömberg and A. Torchinsky, Weighted Hardy Spaces, Lecture Notes in Math. 1381, Springer Verlag, Berlin, 1989.

[SW] J. O. Strömberg and R. L. Wheeden, Fractional integrals on weighted $h^{p}$ and $L^{p}$ spaces, Trans. Amer. Math. Soc. 287 (1985), 293-321.

[Sy] F. A. Sysoeva, Generalization of a certain Hardy inequality, Izv. Vyssh. Ucheb. Zaved. Matematika 49 (1965), No. 6, 140-143 (in Russian).

[VW] I. E. Verbitsky and R. L. Wheeden, Weighted estimates for fractional integrals and applications to semilinear equatuions, J. Funct. Anal. 129 (1995), 221-241.

[W] A. Wedestig, Weighted inequalities of Hardy-type and their limiting inequalities, Doctoral Thesis, Department of Mathematics, Luleå University of Technology, 2003. 